\title{
Los Cuatro Ases de la Baraja: un Trío de Ediciones producidas en Caracas del libro Elementos de Geometría de Legendre más ... una(s) Extraviada(s)
}

\author{
Walter O. Beyer K. \\ nowarawb@gmail.com \\ https://orcid.org/0000-0003-1726-7994 \\ Universidad Nacional Abierta (UNA) \\ Caracas, Venezuela
}

Recibido: 04/04/2020 Aceptado: 08/05/2020

\begin{abstract}
Resumen
Esta investigación tiene como centro el estudio comparativo de tres ediciones venezolanas de los Elementos de Geometría de André Marie Legendre, libro ampliamente usado en Venezuela. La indagación se realizó siguiendo el método histórico y la metodología de análisis de textos históricos. Se dispuso de la existencia de ejemplares de estas ediciones accesibles en las Bibliotecas Nacional de Venezuela, de la Universidad Central de Venezuela y en la personal del investigador. Se hace una descripción del ejemplar de la obra de cada edición, considerando y comparando entre sí diversos elementos constitutivos del texto. Se detalla lo referido a la traducción del impreso, determinándose qué componente nacional estuvo presente y el papel jugado en ello por el ingeniero Jesús Muñoz Tébar. Entre los resultados destacables están: la obra circuló en Venezuela al menos desde 1841 prolongando su presencia hasta bien entrado el siglo XX; la primera edición venezolana disponible es la de 1854: una impresión (sin las notas) de la traducida por Gilmán publicada en París en 1827. La de 1854 es la edición hispanoamericana más antigua hallada hasta ahora; la parte de geometría y la de trigonometría se publicaron en las ediciones analizadas en un solo volumen, siguiendo el patrón de la francesa; en las ediciones consultadas, posteriores a la de 1854, las partes de geometría y de trigonometría tienen diferentes fechas de edición; la edición de 1908/1895 tiene las figuras intercaladas en el texto. Desde 1873 se ha vinculado a Muñoz Tébar con la traducción, pudiendo determinarse qué él es efectivamente el traductor de esta edición del texto y de las subsiguientes tiradas que se hicieron en Venezuela.

Palabras clave: Enseñanza de la Geometría, Legendre, Libros de geometría, Elementos de Geometría

\section{Os quatro ases do baralho: um trio de edições produzidas em Caracas do livro Elementos de Geometría de Legendre e mais ... várias que estão perdidas}

\section{Resumo}

Esta pesquisa enfoca o estudo comparativo de três edições venezuelanas dos Elementos de Geometria de André Marie Legendre, um livro amplamente usado na Venezuela. A investigação foi realizada seguindo o método histórico e a metodologia de análise de textos históricos. Havia cópias disponíveis dessas edições acessíveis na Biblioteca Nacional da Venezuela, na Universidade Central da Venezuela e na pessoal do pesquisador. É feita uma descrição da cópia do trabalho de cada edição, considerando e comparando entre si vários elementos constituintes do texto. É detalhado o que se refere ao a tradução do impresso, sendo determinado qual componente nacional estava presente e o papel desempenhado pelo engenheiro Jesús Muñoz Tébar. Entre os resultados notáveis estão: a obra circulou na
\end{abstract}


Venezuela pelo menos desde 1841, prolongando sua presença até o início do século XX; a primeira edição venezuelana disponível é a de 1854: uma impressão (sem as notas) da traduzida por Gilmán publicada em Paris em 1827, sendo a da 1854 a mais antiga edição hispano-americana encontrada até agora; a parte da geometria e a parte da trigonometria foram publicados nas edições analisadas em um único volume, seguindo o padrão francês; nas edições consultadas, subseqüentes à de 1854, as partes da geometria e da trigonometria têm datas de edição diferentes; a edição de 1908/1895 tem as figuras intercaladas no texto. Desde 1873, Muñoz Tébar está vinculado à tradução, e pode-se determinar que ele é realmente o tradutor desta edição do texto e das edições subsequentes que foram feitas na Venezuela.

Palavras chave: Ensino de geometria, Legendre, Livros de geometria, Elementos de Geometria

\title{
The Four Aces of the Deck of Card: a Trio of Caracas's Editions of the Legendre's book Geometry Elements plus ... several that are lost
}

\begin{abstract}
This research focuses on the comparative study of three Venezuelan editions of the Elements of Geometry by André Marie Legendre, a book widely used in Venezuela. The investigation was carried out following the historical method and the methodology of analysis of historical texts. There were available copies of these editions accessible in the National Library of Venezuela, the library of the Central University of Venezuela and a copy of the researcher. A description of the copy of the book of each edition is made, considering and comparing among themselves various constituent elements of the text. Details regarding the translation of the book are discussed, determining which national component was present and the role played in it by the engineer Jesús Muñoz Tébar. Among the notable results are: the work circulated in Venezuela at least since 1841, prolonging its presence until the beginning of the 20th century; the first Venezuelan edition available is that of 1854: a print (without the notes) of the one translated by Gilman published in Paris in 1827. The 1854 printing is the oldest SpanishAmerican edition found so far; the geometry part and the trigonometry part in the editions analyzed were published in a single volume following the French pattern; in the consulted editions, subsequent to that of 1854, the parts of geometry and trigonometry have different edition dates; the 1908/1895 edition has the figures interspersed in the text. Since $1873 \mathrm{Muñoz}$ Tébar has been linked to translation, and it can be determined that he is indeed the translator of this edition of the text and of the subsequent editions that were made in Venezuela.
\end{abstract}

Keywords: Geometry Teaching, Legendre, books of Geometry, Elements of Geometry

\section{Introducción}

Las obras didácticas llamadas también manuales escolares o libros de texto ocupan un lugar de trascendental importancia dentro del ámbito educativo. Sobre esto es de acotar, como bien señala Gimeno Sacristan (1998) al referirse al nivel curricular que él denomina el curriculum presentado a los profesores, que "el papel más decisivo en este sentido lo desempeñan, por ejemplo, los libros de texto" (p. 124). Al respecto expone Schubring (1987) que "la práctica de la enseñanza no está tan determinada por los decretos ministeriales y los 
planes de estudio oficiales como por los manuales escolares empleados para la enseñanza" (p. 41).

Sin duda alguna uno de tales manuales (o libro de texto) ha sido los Éléments de Géométrie del matemático francés Adrien Marie Legendre (1752-1833) el cual fue empleado por muchos años como libro de estudio para la geometría en muchas partes del mundo, desplazando el uso de los Elementos de Euclides. Particularmente en Venezuela, tanto en su idioma original como traducido al castellano y aún en ediciones autóctonas, este texto dejó su indiscutible impronta.

En virtud de lo antes expuesto se decidió abordar una indagación acerca de la presencia e impacto de los Éléments de Géométrie de Legendre en Venezuela presentando aquí, en este reporte, sólo una parcialidad de los resultados obtenidos: la comparación de tres ediciones venezolanas del libro.

La consulta de fuentes fidedignas como el catálogo de los libreros Damirón y Dopouy (1841) ha permitido determinar que esta obra ya estaba en uso en Venezuela por lo menos desde 1841 ya que ellos lo ofrecían a la venta en dicho año.

Los Éléments ha sido un texto de estudio para la geometría que se instauró en diversos países habiendo tenido traducciones a diversos idiomas. El libro se caracterizó por una amplia difusión teniendo innumerables ediciones, reediciones y reimpresiones así como adaptaciones estando en uso por más de un siglo en varias naciones, incluyendo la nuestra. El conjunto de ediciones y reediciones constituye un complejo entramado que hubo que dilucidar por cuanto en numerosas oportunidades fueron consideradas reediciones y reimpresiones como si fuesen nuevas ediciones de la obra cuando tales tiradas no invulcraron modificaciones, ni en su contenido ni en el formato del libro.

La obra se erigió en un tratado paradigmático catalogado por García Azcárate (2004) como un best-seller, convirtiéndose en el prototipo a seguir y en la guía para muchos autores posteriores. Como ya se indicó, el texto desplazó el arraigado uso que hasta ese momento gozaban los Elementos de Euclides, modernizando la enseñanza de la matemática en esta área. Pero también desplazó a obras contemporáneas competidoras como la de Lacroix.

En virtud de su amplio uso en Venezuela el cual alcanzó hasta el siglo XX, y habiendo habido ediciones venezolanas algunas con traducción autóctona, siguiendo el precepto de Schubring (1987) antes citado se escogió esta obra didáctica para su descripción y análisis en 
lo que se refiere a las ediciones caraqueñas y así a través de ella tratar de comprender, así sea parcialmente, una parcela de una época bastante extensa de nuestra historia pedagógica en lo que a las matemáticas concierne.

En este escrito se va a describir, comparar y analizar la obra considerando ejemplares de tres ediciones venezolanas distintas localizadas en diversas bibliotecas, así como se hará referencia a algunas ediciones realizadas en el país que se conocen sólo por referencias de catálogos u otras fuentes, pero que aún no se han podido localizar ejemplares de las mismas: "las extraviadas".

\section{Algunos elementos teóricos}

Ha de partirse del hecho de que no cualquier impreso, aunque se use dentro de un ambiente escolar, pueda considerarse como una obra didáctica. Así, siguiendo a Beyer (2012) se consideran aquí como obras didácticas, manuales escolares o textos escolares los escritos que sirven de medios auxiliares para el proceso de enseñanza/aprendizaje de una parcela del saber; caracterizados por la presencia de patrones de estructura retórica propios, asociados a un currículo, con un diseño particular y poseedores de un formato y división interna acorde con su finalidad; que exponen una temática extraída de un cuerpo de conocimiento, la cual es transpuesta como saber enseñable y en donde los contenidos han de estar ordenandos considerando simultánea y armónicamente tanto la lógica de la disciplina como ciertos preceptos pedagógicos; en los que la selección de los contenidos obedece a factores de orden educacional, sometidos a fuerzas externas de tipo político, social y económico; escritos que están contextualizados por la cultura de una época específica, con su consiguiente carga ideológica y además presentan valores que dichos impresos acarrean y transmiten; pero, a su vez constituyen un bien económico sujeto a los dictados del comercio.

Por otra parte, es menester tener presente que este estudio trata de una obra la cual es considerada un texto histórico por cuanto fungió en épocas pasadas como libro de enseñanza pero que hoy en día ya ha dejado de serlo por estar en desuso.

La denominación de la obra con el término Éléments tal como aparece en el título original tiene una significación especial. Está asociada con la reforma pedagógica llevada a cabo en Francia después de la Revolución Francesa y en concordancia con ciertos postulados expuestos en la Enciclopedia o Diccionario razonado de las ciencias, las artes y los oficios (L'Encyclopédie o Dictionnaire raisonné des sciences, des arts et des métiers), obra dirigida 
por D’Alembert y Diderot y editada entre 1751 y 1772. A partir de allí se planteó la necesidad de producir libros elementales, obras las cuales debían tomar en consideración los avances discipliarios e ir al meollo de las ciencias, a los aspectos medulares del campo de que se trate; pero debían ser al mismo tiempo comprensibles, didácticas, útiles para la enseñanza. Asimismo estos textos debían ser diferentes a los abregés (resúmenes) que sólo transmitían los rudimentos del campo. En consecuencia, deben distinguirse y no confundirse los elementos y los rudimentos de una ciencia. A ellos se asocia dos tipos de textos con finalidades distintas.

El aspecto antes señalado Schubring (1987) lo considera en su propuesta metodológica para el análisis de libros de texto históricos tomándolo como uno de los patrones a ser tomados en cuenta para dicho ello y que él denomina elementarización y método. Sobre este particular expone que la elementarización tiene que ver con "la transposición del conocimiento [científico] al conocimiento enseñable y un método relacionado" (p. 47). Se trata de un principio similar o asimilable al concepto de Transposición Didáctica propuesto por Chevallard.

Las obras didácticas escritas bajo la concepción de ser libros elementales adoptaron un método de presentación de contenidos característico conocido como método jurídico, el cual desplazó al método catequistico más ajustado para la presentación de los rudimentos y que era frecuentemente empleado en los textos escolares que recibían el nombre de catecismos. El método jurídico es un estilo de redacción por párrafos, los cuales usualmente y en muchas obras didácticas son relativamente cortos y ocasionalmente vienen numerados, guardando semejanza con la presentación de las obras jurídicas (Choppin, 2000; Beyer, 2012). Este tipo de presentación se organiza a veces en una estructura arborescente incluyendo varios subniveles.

La obra aquí estudiada, los Éléments de Géométrie, se redactó siguiendo el método jurídico. Además, está inspirada en la manera formal tradicional, clásica, de presentación de los asuntos matemáticos, conjugándose ambos aspectos.

\section{Metódica}

La presente indagación se ubica dentro del campo de la historia de la educación matemática y es esencialmente de tipo crítico-documental. El método general seguido es el de la investigación histórica (Best, 1961) apoyado en los estudios acerca de textos escolares (Schubring, 1987, 2003; Choppin, 2000). Las fuentes fueron sometidas a la crítica interna y 
externa, privilegiándose las fuentes primarias complementadas éstas con las secundarias cuando ello fuese necesario.

Las unidades de análisis consideradas consistieron en tres ediciones venezolanas del libro Elementos de Geometría de Legendre.

Para la temática aquí tratada, la cual conforma una parcela de una investigación de mayor alcance, el esquema de trabajo partió por determinar en distintas fuentes la presencia de referencias a diversas ediciones venezolanas de los Elementos de Geometría de Legendre, para luego proceder a ubicar la existencia física de ejemplares de algunas de ellas, con la finalidad de garantizar la accesibilidad del material base para su posterior descripción, comparación y análisis. Para este proceso se contó como punto de partida con la posesión por parte del investigador de un ejemplar del texto, editado en Caracas y de algunas fuentes pertinentes al asunto en estudio.

La ulterior búsqueda permitió determinar la presencia de ejemplares de la obra en la Biblioteca de la Universidad Central de Venezuela (UCV), en la Biblioteca Nacional de Venezuela y en la Biblioteca de la Universidad de Los Andes, información que se obtuvo consultando los catálogos digitales de estas dependencias. El arqueo arrojó como resultado la existencia en catálogo de tres (3) ediciones diferentes, una de las cuales aparece en tres bibliotecas. Asimismo, la que posee el investigador está catalogada en una biblioteca (ver Cuadro 1).

Fueron visitadas la biblioteca de la UCV y la Nacional de Venezuela (BNV) para constatar la existencia física de las ediciones catalogadas. Se constató la existencia física de ejemplares, con el plus adicional de que en ambas se localizó un ejemplar de la edición de 1879/1880. Además, en la BNV hay en existencia un ejemplar de la edición 1908/1895, la misma edición del ejemplar que posee el investigador. En total fueron localizados cinco ejemplares (incluyendo el del investigador), correspondientes a tres ediciones distintas. Cada ejemplar consiste en un volumen contentivo de dos obras: la geometría y la trigonometría. Dado que en algunos casos las dos partes que componen la obra tienen fechas de edición distintas usaremos la notación xxxx/yyyy para indicarlo cuando hagamos referencia al volumen como un todo y sólo el año correspondiente si se trata de una parte. Así, p.e. 1908/1895 indica que 1908 corresponde a la Geometría mientras que 1895 refiere a la Trigonometría. Al ser ambas partes editadas en un solo volumen (como se hacía en Francia 
con las ediciones originales) se entiende que la obra como un todo fue publicada en el año más cercano en el tiempo.

En el Cuadro 1 se especifica el material localizado en las bibliotecas.

Cuadro 1: Ejemplares localizados de ediciones venezolanas de los Elementos de Geometría

\begin{tabular}{|c|c|c|c|}
\hline Título & Editor & $\begin{array}{c}\text { Lugar y año de } \\
\text { la edición }\end{array}$ & Ubicación \\
\hline $\begin{array}{l}\text { Elementos de Geometría escritos en francés por A. } \\
\text { M. Legendre, individuo del Instituto de Francia, } \\
\text { de la Real Sociedad de Londres \& c. Traducidos } \\
\text { por la duodécima y última edición } \\
\text { Elementos de Trigonometría escritos en francés } \\
\text { por A. M. Legendre, individuo del Instituto de } \\
\text { Francia, de la Real Sociedad de Londres \& c. } \\
\text { Traducidos por la duodécima y última edición }\end{array}$ & $\begin{array}{l}\text { Imprenta de Juan } \\
\text { de Dios Morales } \\
\text { Imprenta de Juan } \\
\text { de Dios Morales }\end{array}$ & $\begin{array}{l}\text { Caracas, } 1854 \\
\text { Caracas, } 1854\end{array}$ & $\begin{array}{l}\text { Biblioteca } \\
\text { Central } \\
\text { Universidad } \\
\text { Central de } \\
\text { Venezuela } \\
\text { (BUCV) }\end{array}$ \\
\hline $\begin{array}{l}\text { Elementos de geometría (con notas). Escritos en } \\
\text { francés por A. M. Legendre, miembro del Instituto } \\
\text { de Francia, de la Legión de Honor, de la Real } \\
\text { Sociedad de Londres \& c. Traducidos por la } \\
\text { décima y quinta edición. Revisada por el Dr. Jesús } \\
\text { Muñoz Tébar-Ingeniero } \\
\text { Elementos de trigonometría. Escritos en francés } \\
\text { por A. M. Legendre, miembro del Instituto de } \\
\text { Francia, de la Legión de Honor, de la Real } \\
\text { Sociedad de Londres \& c. Traducidos por la } \\
\text { décima y quinta edición. Revisada por el Dr. Jesús } \\
\text { Muñoz Tébar-Ingeniero }\end{array}$ & $\begin{array}{l}\text { Alfred Rothe- } \\
\text { Editor }\end{array}$ & Caracas, 1879 & $\begin{array}{l}\text { BUCV } \\
\text { Biblioteca } \\
\text { Nacional de } \\
\text { Venezuela } \\
\text { (BNV) } \\
\text { Biblioteca } \\
\text { Universidad } \\
\text { de Los } \\
\text { Andes } \\
\text { (BULA) }\end{array}$ \\
\hline $\begin{array}{l}\text { Elementos de geometría (con notas). Escritos en } \\
\text { francés por A. M. Legendre. Miembro del Instituto } \\
\text { de Francia, de la Legión de Honor, de la Real } \\
\text { Sociedad de Londres, etc. Última edición. Figuras } \\
\text { intercaladas en el texto. Revisada por el Dr. Jesús } \\
\text { Muñoz Tébar-Ingeniero } \\
\text { Elementos de trigonometría. Escritos en francés } \\
\text { por A. M. Legendre. Miembro del Instituto de } \\
\text { Francia, de la Legión de Honor, de la Real } \\
\text { Sociedad de Londres, etc. Última edición. Figuras } \\
\text { intercaladas en el texto. Revisada por el Dr. Jesús } \\
\text { Muñoz Tébar-Ingeniero }\end{array}$ & $\begin{array}{l}\text { L. Puig Ros y } \\
\text { Hermano }\end{array}$ & Caracas, 1908 & $\begin{array}{l}\text { Biblioteca } \\
\text { personal del } \\
\text { investigador }\end{array}$ \\
\hline
\end{tabular}

Fuente: Elaboración propia basada en Legendre (1854a, 1854b, 1879, 1880, 1895, 1908)

Una vez verificada la existencia de dichos ejemplares se procedió a diseñar un esquema para la adecuada descripción, comparación y análisis del material en existencia.

Los elementos principales escogidos para ser objeto de comparación y/o análisis fueron esencialmente: el título de la obra (en extenso) y demás datos editoriales; la descripción física (dimensiones, formato, paginación); la división interna y el ordenamiento de las partes y 
subpartes del volumen; el texto de la advertencia; la redacción de la primera página de cada libro y de las notas, así como la ubicación de las figuras. Adicionalmente se consideraron otros elementos presentes en la obra.

Se extrajeron los datos y la información pertinente antes indicada de cada uno de los ejemplares consultados. Asimismo, en cada caso se tomaron una serie de notas manuscritas acerca de otros detalles y datos relevantes de cada libro. Buena parte de dicha información fue vaciada en un conjunto cuadros y tablas a los fines de poder hacer comparaciones y luego proceder a su discusión y análisis. Como instrumento auxiliar para la recolección de información se empleó la cámara de un teléfono celular para tomar fotografías de ciertas páginas relevantes de los impresos.

A efectos de contrastación se procedió a localizar en Internet versiones digitales de las ediciones francesas originales $12^{\mathrm{a}}$ y $15^{\mathrm{a}}$ (Legendre, 1823, 1862), de las cuales proceden las traducciones que corresponden a las ediciones encontradas, así como de ediciones de la obra modificada por Blanchet.

Después de tener a mano los elementos descriptivos y otra información relevante se procedió a contrastarla y analizarla para así poder arribar a un conjunto de conclusiones acerca de las características físicas, estructura interna y enfoque del texto en estudio, así como del origen y autoría de sus traducciones.

\section{Descripción física, datos editoriales y estructura de los ejemplares de la obra}

Las dimensiones físicas de los cinco ejemplares revisados se muestran en la Tabla 1.

Tabla 1

Dimensiones de los ejemplares consultados

\begin{tabular}{crr}
\hline Edición & \multicolumn{2}{c}{ Dimensiones } \\
\hline Edición 1854/1854 & $20,5 \times 13,5 \mathrm{~cm}$ & \multicolumn{1}{c}{ (BUCV) } \\
Edición 1879/1880 & $22,5 \times 14,2 \mathrm{~cm}$ & (BUCV) \\
Edición 1879/1880 & $22,5 \times 14,5 \mathrm{~cm}$ & $(\mathrm{BNV})$ \\
Edición 1908/1895 & $21 \times 14,5 \mathrm{~cm}$ & (Personal) \\
Edición 1908/1895 & $21 \times 14,2 \mathrm{~cm}$ & $(\mathrm{BNV})$ \\
\hline
\end{tabular}

Fuente: Elaboración propia

Las dimensiones anteriores corresponden al formato que los bibliógrafos, editores y libreros clásicamente denominan en $8^{\circ}$, el mismo que usaron los editores europeos. 
Un elemento a ser destacado es la encuadernación de todos los ejemplares revisados la cual es del tipo tapa dura. El ejemplar de la edición 1908/1895 de la BNV así como el de la edición 1854/1854 de la BUCV están restaurados y sus encuadernaciones no son las originales. En la tapa o cubierta de los ejemplares, salvo el de 1854/1854, no aparece ninguna indicación sobre la obra pero todas están identificadas en el lomo.

Los respectivos títulos son extensos y descriptivos, muy al estilo de la época, incluyéndose allí una especie de microcurrículo del autor, así como cierta referencia sobre la traducción y la base de la misma.

Las diferentes ediciones del texto consultadas (las ediciones: 1854/1854, 1879/1880, 1908/1895) estaban integradas por dos escritos o partes independientes: Elementos de Geometría y Elementos de Trigonometría, impresas en un solo volumen. Adicionalmente existen unas notas a la parte de geometría las cuales se omiten en la edición de 1854/1854. En las distintas ediciones del texto, después de la portada de la parte referida a trigonometría se encuentra en una portadilla el título Tratado de Trigonometría, presentándose así un cambio de denominación con respecto a la de la portada (Elementos de Trigonometría).

Se inicia cada ejemplar con una Advertencia la cual ocupa dos páginas. En la edición de 1854/1854 es la traducción de la Advertencia presente en la edición francesa de 1823 (la $12^{\circ}$ de Legendre). En las otras ediciones es una traducción de su similar que aparece en la $15^{\mathrm{a}}$ edición parisina de 1862 .

Cuadro 2: Constitución de cada volumen y extensión de sus componentes

\begin{tabular}{|c|c|c|c|c|c|}
\hline $\begin{array}{c}\text { Año(s) de la } \\
\text { edición }\end{array}$ & Advertencia & $\begin{array}{l}\text { Cuerpo de los } \\
\text { Elementos de } \\
\text { Geometría }\end{array}$ & Notas & $\begin{array}{l}\text { Cuerpo de los } \\
\text { Elementos de } \\
\text { Trigonometría }\end{array}$ & Figuras \\
\hline $1854 / 1854$ & pp. 5-6 & No hay & pp. 5-92 & $\begin{array}{c}\text { Al final: } \\
\text { láminas } \\
\text { desplegables }\end{array}$ \\
\hline $1879 / 1880$ & pp. 5-6 & pp. 7-147 & $\begin{array}{c}\text { pp. 53-92 } \\
\text { (siguen a la } \\
\text { Trigonometría) }\end{array}$ & pp. 3-50 & $\begin{array}{l}\text { Al final: 14 } \\
\text { láminas } \\
\text { desplegables }\end{array}$ \\
\hline $1908 / 1895$ & pp. 5-6 & pp. 7-251 & pp. 252-314 & pp. 3-77 & $\begin{array}{l}\text { Intercaladas en } \\
\text { el texto }\end{array}$ \\
\hline
\end{tabular}

Fuente: Elaboración propia basada en Legendre (1854a, 1854b, 1879, 1880, 1895, 1908)

Como se desprende del Cuadro 2 las dos obras que conforman cada volumen tienen paginación independiente. Además, como ya se advirtió con anterioridad, los años de edición que aparecen en las respectivas portadas de cada parte no necesariamente son coincidentes. En razón de lo cual hay que colegir que la parte de la obra con fecha editorial más antigua fue 
simplemente reimpresa y como fecha de edición de todo el volumen hay que asumir la más cercana en el tiempo.

Otra característica que puede observarse en el Cuadro 2 es que en la tirada de 1854 fueron omitidas las notas, las cuales sí aparecen en las otras dos ediciones consultadas aunque distintamente ubicadas; en las ediciones 1854/1854 y 1879/1880 las figuras venían siendo agregadas al final en láminas desplegables, mientras que en la edición de 1908/1895 ahora están intercaladas en el texto.

Un detalle a considerar es que los ejemplares revisados carecen de índice y de privilegio.

Las diferencias en el número de páginas son explicables en parte a cambios en las dimensiones de la mancha del volumen (la edición de 1854/1854 tiene una mancha ligeramente menor) y en el caso de la de 1908/1895 debido a que las figuras ahora están intercaladas en el texto lo que cambia el diseñö del libro.

La parte de geometría se divide en ocho (8) libros, siguiendo la tradición euclidea. Los temas tratados discriminados por libro son los siguientes:

Cuadro 3: División interna de la parte de geometría y su temario

\begin{tabular}{|c|c|l|}
\hline LIBRO & EDICIÓN & \multicolumn{1}{|c|}{ TEMARIO } \\
\hline I & $1854,1879,1908$ & Principios \\
\hline II & $1854,1879,1908$ & Del círculo y de la medida de los ángulos \\
\hline III & $1854,1879,1908$ & Proporcionalidad de las figuras \\
\hline IV & $1854,1879,1908$ & De los polígonos regulares y de la medida del círculo \\
\hline $\begin{array}{c}\text { Apéndice } \\
\text { al Libro } \\
\text { IV }\end{array}$ & 1854 & No aparece \\
\cline { 2 - 3 } & 1879 & Sin título). Contiene definiciones y teoremas \\
\hline V & 1908 & No aparece \\
\hline VI & $1854,1879,1908$ & De los planos y ángulos sólidos \\
\hline VII & $1854,1879,1908$ & Los poliedros \\
\hline $\begin{array}{c}\text { Apéndice } \\
\text { a los } \\
\text { Libros VI } \\
\text { y VII }\end{array}$ & $1854,1879,1908$ & La esfera \\
\hline VIII & & De los poliedros regulares \\
\hline
\end{tabular}

Fuente: Elaboración propia basada en Legendre (1854a, 1879, 1908)

La denominación de los ocho libros que conforman la parte de geometría es idéntica en las tres ediciones. Con respecto a los apéndices, el correspondiente al Libro IV sólo está en la 
edición de 1879/1880; mientras que el referido a los Libros VI y VII aparece en las tres ediciones consultadas.

A continuación mostramos la extensión de cada uno de los ocho (8) libros que componen el escrito referido a geometría.

Cuadro 4: Extensión (en páginas) de los libros que componen los Elementos de Geometría

\begin{tabular}{|c|c|c|c|c|c|c|c|c|c|c|}
\hline $\begin{array}{c}\text { Edición } \\
\text { de la Geo. }\end{array}$ & $\begin{array}{c}\text { Libro } \\
\text { I }\end{array}$ & $\begin{array}{c}\text { Libro } \\
\text { II }\end{array}$ & $\begin{array}{c}\text { Libro } \\
\text { III }\end{array}$ & $\begin{array}{c}\text { Libro } \\
\text { IV }\end{array}$ & $\begin{array}{c}\text { Apén- } \\
\text { dice }\end{array}$ & $\begin{array}{c}\text { Libro } \\
\text { V }\end{array}$ & $\begin{array}{c}\text { Libro } \\
\text { VI }\end{array}$ & $\begin{array}{c}\text { Libro } \\
\text { VII }\end{array}$ & $\begin{array}{c}\text { Apén- } \\
\text { dice }\end{array}$ & $\begin{array}{c}\text { Libro } \\
\text { VIII }\end{array}$ \\
\hline 1854 & $7-26$ & $27-43$ & $44-74$ & $74-88$ & ------ & $89-104$ & $104-128$ & $129-145$ & $146-150$ & $150-169$ \\
\hline 1879 & $7-23$ & $24-37$ & $38-62$ & $62-74$ & $74-77$ & $78-90$ & $91-110$ & $111-127$ & $127-130$ & $131-147$ \\
\hline 1908 & $7-36$ & $37-65$ & $66-111$ & $112-133$ & ------- & $134-155$ & $156-190$ & $191-215$ & $216-222$ & $223-251$ \\
\hline
\end{tabular}

Fuente: Elaboración propia basada en Legendre (1854a, 1879, 1908)

\section{Los editores venezolanos del libro}

El primer comercio nacional que conocemos ofreciendo la obra es la empresa editorial de Damirón y Dupouy. Esta información se extrajo de su catálogo de 1841 mediante el cual es publicitado el texto para su venta. Sería de sumo interés localizar un ejemplar de esta edición para realizar un análisis detallado del mismo y poder dilucidar si la edición y/o producción física (impresión) se realizó o no en Venezuela. Si fuese edición autóctona sería notable dados los requerimientos técnicos exigidos para imprimir un libro de matemáticas en aquel entonces. Es este el momento de un arranque más firme en la producción criolla de textos referidos a las ciencias exactas (ver Beyer, 2012). Se hallaba localizada esta casa editora en la esquina de Sociedad y su catálogo parece ser uno de los primeros de su tipo que circularon en Venezuela (Castellanos, 2017a).

Posteriormente se encuentra la edición del texto de 1854 debida al impresor Juan de Dios Morales. Es una de las que se localizó a efectos de esta investigación. En 1855 el comercio la Canastilla de los Estudiantes ofrece al público un conjunto de libros entre los cuales se encuentra la obra de Legendre en edición caraqueña (Castellanos, 2017a).

En años subsiguientes hay nuevas ediciones que no hemos podido localizar las cuales forman parte del conjunto de "las extraviadas". Así, por ejemplo, para 1873 se encuentra un aviso en La Opinión Nacional (citado por Castellanos, 2017b, p. 67) ofreciendo una edición nacional traducida “de la $15^{\mathrm{a}}$ Edición. Revisada por el Dr. Muñoz Tébar”, coligadas a tal fin la Librería de Rojas Hermanos y la Librería de Alfred Rothe. Esta es la primera mención a Muñoz Tébar asociándolo con el libro. 
Una casa editora de renombre, la de Alfred Rothe, publicó nuevamente el libro de Legendre con fecha de edición 1879/1880. En la Portada de esta tirada del libro se indica que fue traducida de la décima quinta edición (la francesa de 1862) y revisada por el ingeniero venezolano Jesús Muñoz Tébar.

Se encuentran referencias a otras ediciones (Beyer, 2019) entre las que cabe destacar la realizada en el siglo XX por la casa editora de L. Puig Ros (edición 1908/1895), empresa que aún en 1944 mantenía el libro en catálogo. Es la edición que posee el investigador.

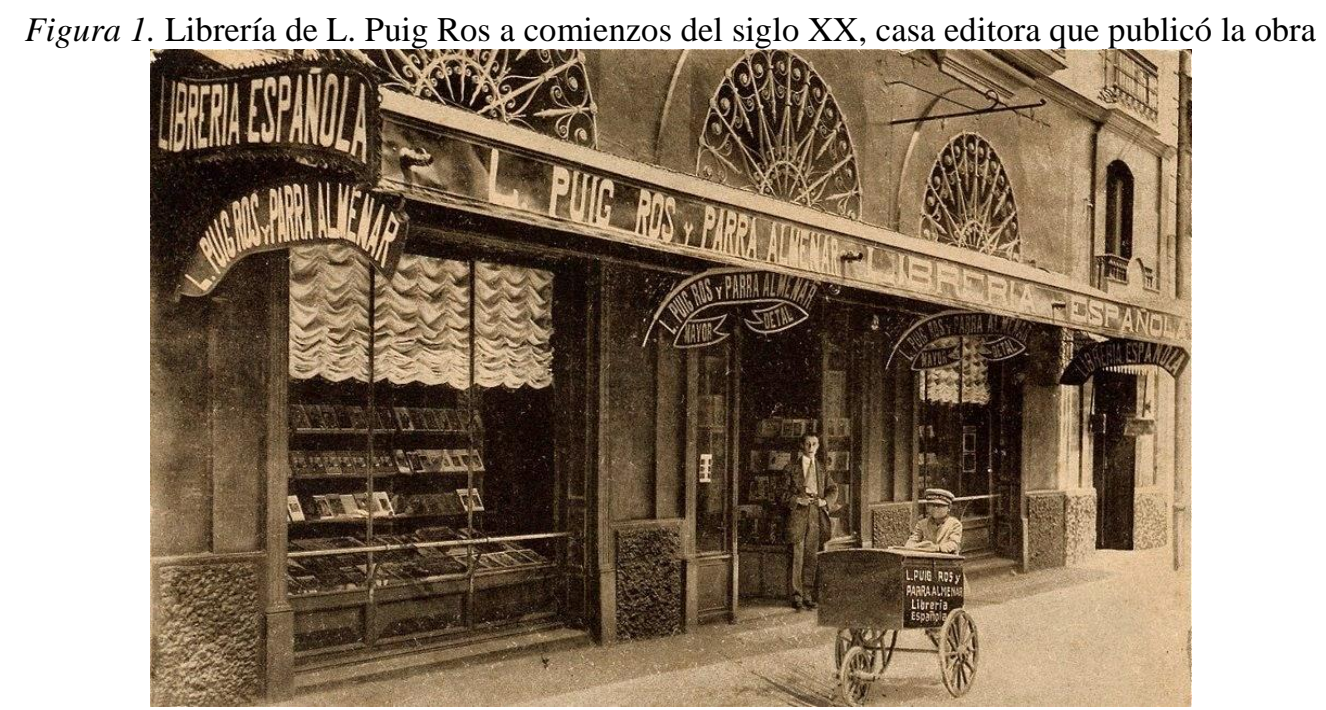

Fuente: Museo del Transporte Guillermo José Schael (2014).

\section{Comparación y análisis de tres ediciones venezolanas distintas de la obra}

Obra y autor son un binomio indisoluble y su análisis hay que encuadralo dentro de las estrechas relaciones entre ambos y considerando los diversos contextos que les son pertinentes.

El proceso de detección de ediciones venezolanas de la obra condujo a constatar un conjunto de éstas que abarca un período de tiempo que corre desde 1854 hasta el siglo XX, ediciones las cuales se encuentran reseñadas en diversas fuentes. En dicho lapso fueron diversas las casas editoras que se ocuparon de publicar el libro; pero, a pesar de ello la existencia física de ejemplares es escasa y apenas se pudo encontrar los correspondientes a kas tres ediciones antes señaladas (ver Cuadro 1).

Las tres ediciones localizadas que se analizan son las de 1854/1854, 1879/1880 y 1908/1895 cuyas portadas se muestran en la Figura 2. 
Figura 2. Portadas de las tres ediciones venezolanas analizadas de la geometría de Legendre: 1854, 1908 y 1879
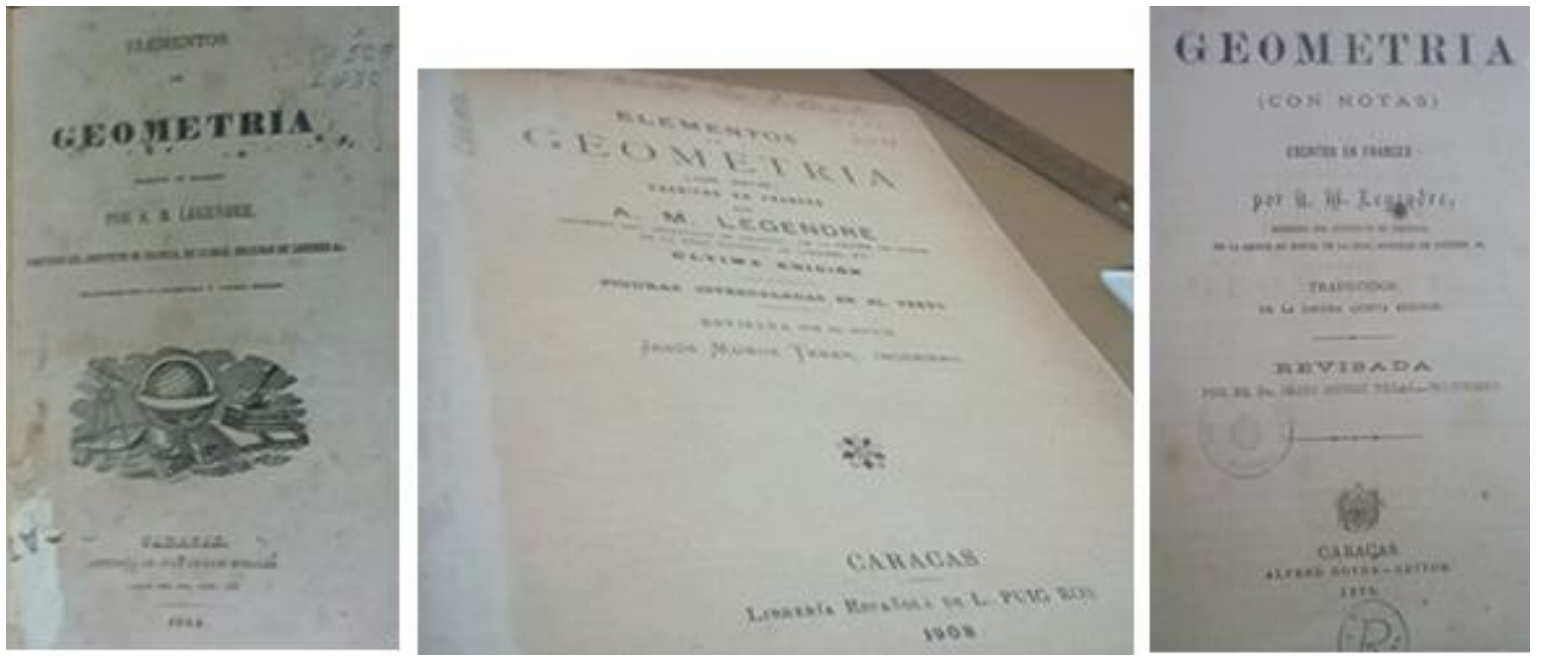

Fuente: Elaboración Propia

Al comparar los ejemplares de las tres ediciones se pudieron observar algunos hechos notorios. De seguidas destacamos algunos.

La edición de 1908/1895, a diferencia de las otras, trae las figuras intercaladas en el texto, lo cual se anuncia ya en su portada. Las figuras aparecen numeradas (con números indoarábicos) correlativamente a lo largo del impreso.

En todas las ediciones el texto en su parte de geometría se subdivide en 8 libros siguiendo la tradición euclidea (ver Cuadro 3). A su vez cada uno de los libros se organiza iniciando con las Definiciones seguidas de un conjunto de apartados o secciones titulados Proposiciones numerados en forma independiente en cada libro; éstas últimas incluyen teoremas, lemas, escolios, corolarios y problemas (no siempre aparecen todos estos elementos). Adicionalmente en algunos libros (II-IV) se contempla al final una sección separada de problemas. Ocasionalmente encontramos al final de un libro una sección denominada Escolio general. Adicionalmente, en el Libro I se proporciona una explicación de los términos y signos empleados y se enuncian cinco axiomas siguiendo a Euclides.

La parte de trigonometría también se divide en secciones pero éstas carecen de numeración. Es de recordar que aunque la trigonometría aparece en conjunto con la geometría en un mismo volumen sin embargo es un texto independiente de la ésta lo cual explica la diferencia de diseño en la estructuración del escrito y el tener una paginación propia. 
El estilo de presentación del contenido sigue básicamente el modelo jurídico el cual se adapta a la formalidad matemática del escrito.

Se pudo observar que en la Geometría editada en 1908 la extensión de cada Libro es mucho mayor que en las precedentes. Una razón para ello es que ahora las figuras aparecen intercaladas en el texto y no al final de la obra en láminas desplegables como solían estar en ediciones previas. Asimismo, ocurre con la parte asociada a la Trigonometría. Por otro lado, comparando la mancha de esta edición con la de la edición de 1879 la última es mayor, por lo tanto hay más texto por página que en la primera.

Se observaron algunos llamados a pie de página en la edición de 1908 los cuales remiten a las notas.

En la comparación de algunos pasajes se han encontrado leves diferencias de redacción entre una y otra edición.

Cuadro 5: Ejemplos de pequeñas diferencias de redacción entre las ediciones

\begin{tabular}{|c|l|}
\hline Edición & \multicolumn{1}{c|}{ Muestra de texto con algunos cambios } \\
\hline 1854 & $\begin{array}{l}\text { "I La Geometría es una ciencia que tiene por objeto la medida de la extensión. La extensión tiene } \\
\text { tres dimensiones, longitud, latitud y altura ó profundidad" (Legendre, 1879, p. 7) }\end{array}$ \\
\hline 1879 & $\begin{array}{l}\text { "I La Geometría es una ciencia que tiene por objeto la medida de la extensión. } \\
\text { La extensión tiene tres dimensiones, longitud, latitud y altura" (Legendre, 1879, p. 7) }\end{array}$ \\
\hline 1908 & $\begin{array}{l}\text { "I La Geometría es una ciencia que tiene por objeto la medida de la extensión de las figuras, y el } \\
\text { estudio de sus propiedades. } \\
\text { La extensión tiene tres dimensiones, longitud, latitud y altura ó profundidad" (Legendre, 1908, p. } \\
7)\end{array}$ \\
\hline
\end{tabular}

Fuente: Elaboración propia basada en Legendre (1854a, 1879, 1908)

Aquí se han resaltado con cursivas las pequeñas diferencias (omisión/agregados) en las ediciones correspondientes a 1854 y 1908 con respecto a la de 1879. Dichas modificaciones en las ediciones de 1879 y 1908 han de atribuirse al trabajo realizado por Muñoz Tébar. Cotejando este extracto con su correspondiente en las ediciones francesas $12^{\mathrm{a}}$ y $15^{\mathrm{a}}$ se encuentra que en la versión de 1879 se presenta una traducción literal mientras que en la de 1908 se agregan algunas palabras que aclaran el contenido.

Otra diferencia es el Apéndice al libro IV incluido en la edición de 1879 el cual está ausente en las ediciones de 1854 y 1908, modificaciones atribuibles a Muñoz Tébar. Asimismo en la edición de 1879/1880 las notas están luego de la parte de trigonometría paginadas correlativamente con ésta (ver resaltado en el Cuadro 2), mientras que en la de 1908/1895 éstas están intercaladas entre la geometría y la trigonometría. Por su lado la edición de 1854/1854 carece de dichas notas. 


\section{Una discusión acerca de la(s) traducción(es) editada(s) en Venezuela}

Un elemento importante a ser considerado es el referido a las traducciones de la obra. A tal efecto se consideró la evolución editorial del libro fuera de nuestro país y la historia editorial del mismo dentro de nuestras fronteras, así como las traducciones castellanas del texto. Para esto nos basamos en varios estudios (García Azcárate, 2004; Schubring, 2009; Beyer, 2019).

Es de interés anotar que Albis González (1977) y Albis G. y Sánchez B. (s/f) reportan la existencia de una traducción de la versión modificada por Blanchet, realizada por Lleras en Colombia y publicada en 1866, la cual ha sido considerada como la primera de su tipo en América Latina. Por su lado, García Azcáte (2004) hace mención a las dos traducciones realizadas por el español Gilman en 1807 y 1827, respectivamente.

Al estar registrada la circulación en Venezuela de versiones de la obra en castellano desde 1841 es de interés determinar su procedencia y quién las tradujo. Esto se complica dado que de varias de tales ediciones no se han podido obtener ejemplares ("las extraviadas") y sólo es dable establecer algunas conjeturas sobre éstas.

La primera edición caraqueña que puede documentarse sale a la luz en 1854 publicada por Juan de Dios Morales. Antecede pués esta edición a la bogotana de 1866 teniendo en consecuencia la venezolana la primacía; es decir, es ésta la primera edición de la obra de Legendre en tierras americanas. Cabe entonces preguntarse si también corresponde a una traducción realizada en Venezuela.

La respuesta a la anterior interrogante es negativa. El análisis del ejemplar consultado indica que se trata de una traducción de la $12^{a}$ edición francesa del libro original de Legendre el cual fue publicado en 1823, justamente la que realizó Gilman en 1827. Para dicho análisis se consideró lo afirmado por el traductor en la Advertencia de la obra y el estudio realizado por García Azcárate (2004) lo cual permitió contrastar algunos elementos del ejemplar consultado con sus análogos de la traducción de Gilman citados por esa investigadora.

Las posteriores ediciones caraqueñas que han podido detectarse, la de Rojas Hermanos de 1865 y la de Alfred Rothe de 1868, tienen todas las características de ser nuevamente tiradas de la traducción de Gilman de 1827 de acuerdo con la información recopilada. Refieren que son traducción de $12^{\circ}$ edición, la francesa de 1823 . No ha podido consultarse ningún 
ejemplar de las mismas. Vale decir son nuevas tiradas del mismo libro que fue publicado en 1854 por Juan de Dios Morales.

Una subsiguiente edición, la de 1873, se encuentra anunciada en el periódico La Opinión Nacional (citado por Castellanos, 2017b, p. 67) en donde se ofrece una edición nacional traducida de la "15 a Edición. Revisada por el Dr. Muñoz Tébar [cursivas añadidas]". Se asociaron para esta tarea la Librería de Rojas Hermanos y la Librería de Alfred Rothe. Evidentemente se trata de una nueva traducción de la obra basada en una versión original distinta a la empleada por Gilman, quien lo había hecho en 1827 sobre la base de la $12^{\mathrm{a}}$ edición francesa de 1823, mientras que ésta se hacía de la $15^{\mathrm{a}}$ edición francesa de 1862 .

Es ésta la primera mención explícita vinculando a Muñoz Tébar con el libro. Llama la atención que allí no se afirme directamente que él la tradujo y se le adjudique sólo el papel de revisor.

Alfred Rothe imprime nuevamente el texto (edición de 1879/1880) con la coletilla “Traducidos de la décima quinta edición. Revisada por el Dr. Jesús Muñoz Tébar, Ingeniero [cursivas añadidas]” (Legendre, 1879, Portada). Así la reportan también los catálogos de las bibliotecas y bibliógrafos como Sánchez (1946). Es una de las analizadas en este escrito.

Con respecto a esta edición de 1879/1880 de los Éléments el acucioso investigador colombiano Albis González (1977) expresa que la traducción "fue realizada en Venezuela bajo la supervisión de Jesús Muñoz Tébar en 1879 [cursivas añadidas]" (p. 339) y en la ficha de la obra que él anexa separa la Geometría, la Trigonometría y las Notas, afirmando que Muñoz Tébar supervisó las traducciones de las dos primeras partes mientras que con respecto a las notas indica que éstas fueron "Traducidas de la décima quinta edición francesa por el doctor Jesús Muñoz Tevar (sic) Ingeniero [cursivas añadidas]” (op. cit., p. 340).

Puede evidenciarse que existen discrepancias y versiones contrapuestas sobre este asunto. Así, biógrafos y otros estudiosos aseveran que Muñoz Tébar sí fue el traductor de todo el libro. Se tiene que Pardo Stolk y de Amezaga (1973), Olivar (2008), Cilento Sarli et al. (1999) y Freytes (2000) así lo afirman tajantemente.

Otra fuente, la copia facsimilar de la Memoria del Ministerio de Obras Públicas de 1875 editada en 1975 para celebrar el centenario de la creación de dicho ministerio, tiene un estudio preliminar realizado por los editores en donde se afirma que Muñoz Tébar “dejó prueba impresa [de su inclinación por los estudios matemáticos] en sus traducciones de los 
libros de Lacroix y de Legendre sobre álgebra y geometría ... [cursivas añadidas]” (p. 12). Hay aquí nuevamente una afirmación categórica sobre este asunto.

También Frydensberg (1895) en su catálogo de obras didácticas de aquella época en referencia a la participación de Muñoz Tébar coloca: "Elementos de geometría por A. M. Legendre, traducidos al castellano y arreglados según la 14ª edición" (p. 331), atribuyéndole a Muñoz Tébar la labor de traducción aunque la asocia con la $14^{\circ}$ francesa y no con la $15^{\mathrm{a}}$. Un editor del libro en un catálogo inserto en otra obra (Rothe, 1880) también señala a Muñoz Tébar como traductor.

Resumiendo lo antes expuesto aparecen dos señalamientos: en uno se expresa que Muñoz Tébar tradujo la obra (íntegramente) y en el otro se indica que tradujo las notas de la décima quinta edición y/o revisó (o mejoró) la traducción del cuerpo de la obra.

Para dirimir este asunto se requiere realizar un cuidadoso análisis. Se comenzará por bosquejar quién era Jesús Muñoz Tébar enfatizando en su formación profesional, su experiencia y sus inclinaciones.

El insigne ingeniero venezolano Jesús Muñoz Tébar (1847-1909) “culmina satisfactoriamente [en 1866] el tercer bienio en la Academia Militar de Matemáticas de Caracas y obtiene el grado de Teniente de Ingenieros. Para entonces ya había recibido el título de Bachiller en Filosofía en la Universidad Central de Venezuela" (Olivar, 2008, p. 27). Además dominaba el idioma francés.

Es de destacar que Muñoz Tébar profesaba un gran amor por las ciencias exactas a lo cual debemos sumarle el hecho de que "en 1868 es designado profesor del primer bienio de la Academia de Matemáticas y en 1870 aparece dictando cátedra a los alumnos del tercero" (Olivar, 2008., p. 29). Asimismo en 1872 dicta clases en el $2^{\circ}$ bienio. Adicionalmente en ese año dirige una importante escuela experimental y su afición por la geometría y su dedicación a la docencia lo llevaron a escribir varias obras didácticas (Catecismo del Sistema Métrico Decimal, para el uso de las escuelas de la República, 1873; Jeometría para el uso de las escuelas de la República, 1877).

Hay que resaltar que en dicha Academia se empleaba desde sus inicios en 1831 los Éléments como texto de estudio para la geometría. Este hecho se deduce de las propias palabras de su Director, Juan Manuel Cagigal, quien en su informe de 1838 declara haber incorporado a la naciente biblioteca "algunas de las obras que sirven de texto de la escuela 
militar de West Point" (Cagigal, 1838, p. 37); expresando además que en en dicho plantel "se enseña [...] por los mismos textos adoptados en la escuela de puentes y calzadas de Francia" (Cagigal, 1838, p. 48). En ambas instituciones extranjeras era obligado el uso del libro de Legendre (Preveraud, 2013; École des Ponts et Chaussées, 1872).

Asimismo, Zawisza (1980) habla de "la modesta biblioteca completada por Cagigal" (p. 61) señalando que “en 1835 llegan los libros de Francia [...]” (Zawisza, 1988, p. 174), dotación que ya reportaba Cagigal en su tercer y cuarto informes (Cagigal, 1834, 1836). Adicionalmente Zawisza (1980) reporta que en un lote de libros adquiridos por la Academia entre 1865-1866 vino la versión Éléments de géométrie avec additions et modifications par Blanchet la cual correspondía a la $2^{\mathrm{a}}$ edición basada en la $15^{\mathrm{a}}$ de Legendre (Deuxiéme Édition suivie de la Quinziéme Edition). También da noticia de la presencia en la biblioteca del plantel en el año 1866 de otra edición de la obra traducida al castellano. Ernst (1875) y Zawisza (1980) afirman que allí también estaba la 12a edición original en francés (la de 1823).

Lo antes señalado indica el uso continuado en esta institución del libro de Legendre encontrándose allí diversas ediciones tanto en francés como en castellano. En consecuencia Muñoz Tébar como estudiante y docente de la Academia manejaba los contenidos presentados en la obra y tuvo la oportunidad de tener a la mano distintas versiones de la misma. Asimismo pudo percibir deficiencias en la traducción de Gilman y omisiones como la de las notas en la edición caraqueña de 1854. Tenía pués Muñoz Tébar la motivación y todas las credenciales a su favor para abordar la labor de traductor la cual emprendió vertiendo los Éléments al idioma de Cervantes.

Al cotejar la edición de 1854/1854 con la de 1879/1880 (cuya traducción se le atribuye a Muñoz Tébar) se constata que existen cambios de redacción así como en ella están incorporadas las notas las cuales están ausentes en la edición de 1854.

Como ya se dijo, Muñoz Tébar debió percatarse de algunas cuestiones no satisfactorias en las versiones castellanas de Gilmán y le parecería pertinente mejorarlas, pero podría también haber sido un encargo del editor o ambas circunstancias la motivación para emprender esta difícil tarea.

Muñoz Tébar podía disponer de las traducciones al castellano de Gilman que habían circulado en el país y contaba con las ediciones francesas: la $12^{\mathrm{a}}$ y la $15^{\mathrm{a}}$ de París (Legendre, 
1823, 1862) para cotejar aquellas, así como la versión modificada de Blanchet. Tal vez incluso tuvo acceso a la traducción colombiana de 1866.

Los cambios de redacción encontrados al cotejar la edición de 1854/1854 con la 1879/1880 constituyen un claro indicativo de que Muñoz Tébar además de traducir las notas también iba traduciendo el libro basándose en la $15^{\mathrm{a}}$ edición, teniendo a la vista las versiones españolas de Gilman, haciéndole modificaciones, ajustes y mejoras al trabajo del traductor ibérico. La incorporación en 1879 y subsecuente desincorporación en 1908 del Apéndice al libro IV hay que achacársela al ingeniero venezolano. Esa es la manera usual de trabajo de un traductor cuando existe(n) traducción(es) previa(s) de una obra.

Finalmente, en el título de la edición 1908/1895 ya no se habla de traducción sino sólo de revisión lo cual es indicativo que para esta tirada Muñoz Tébar se limitó a revisar su trabajo previo de traducción, haciéndole ajustes y mejoras.

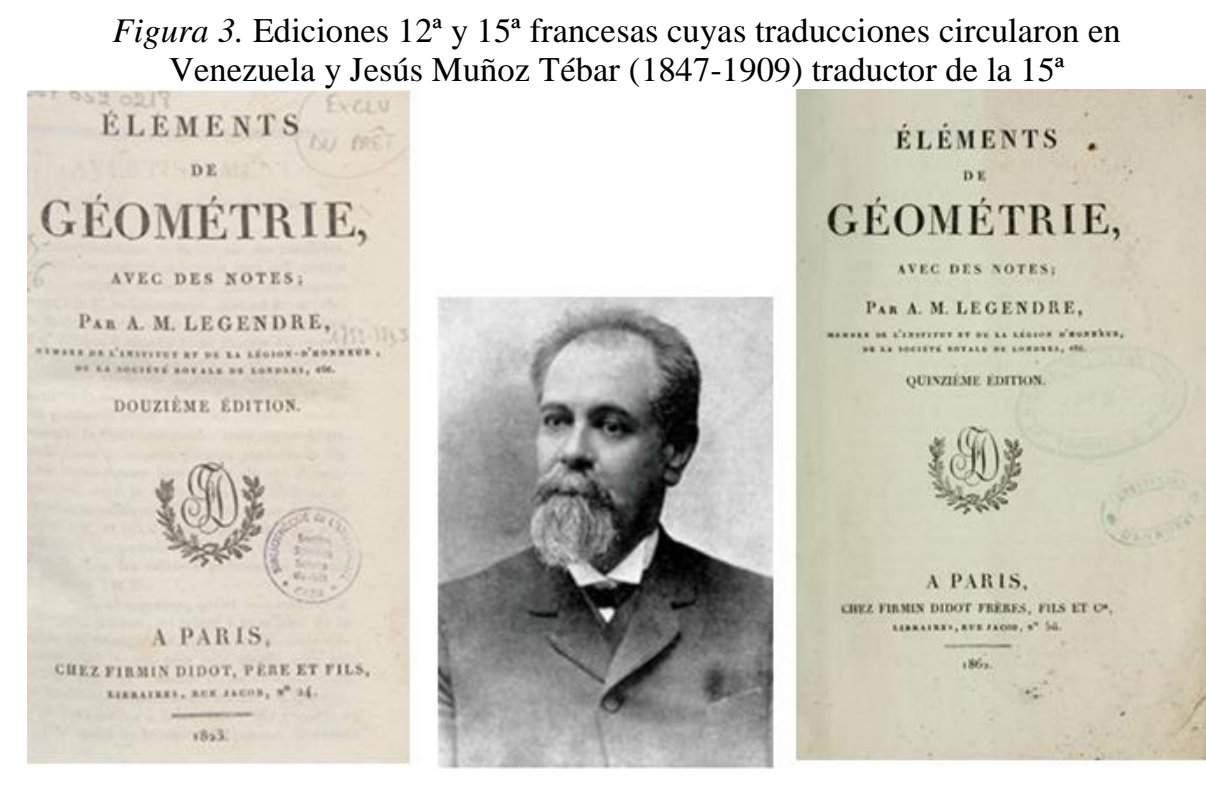

Fuente: diversas páginas de Internet

\section{Algunos resultados y conclusiones referidos a las ediciones venezolanas}

Los datos e información recopilados y su posterior análisis conducen a un conjunto de conclusiones que se expondrán en esta sección.

Un primer hecho a ser señalado es que existe suficiente documentación que permite estudiar con cierta precisión la obra y la evolución histórica de las ediciones venezolanas, principalmente a través de los catálogos de las empresas editoras y aquellos realizados por diversos bibliógrafos. Además distintas investigaciones y ensayos vinculados con la temática 
educativa y con la bibliografía nacional orientan en torno a la existencia de diversas ediciones (foráneas y nacionales) de los Elementos de Geometría de Legendre, aunque no todas estas ediciones están disponibles en las bibliotecas. Asimismo, durante la realización de la presente investigación se ha podido constatar la existencia física de ejemplares de tres ediciones del libro en distintas bibliotecas del país.

La indagación ha conducido a determinar fehacientemente la presencia del texto en el mercado librero nacional por lo menos desde 1841. Asimismo, la documentación basada en fuentes primarias permite aseverar la permanencia continuada de la obra en dicho mercado durante el resto del siglo XIX hasta bien entrado el siglo XX, mediante la importación de ediciones foráneas (traducidas al castellano y en francés) así como mediante la producción de diversas ediciones nacionales, incluso algunas traducidas en el país.

Este texto se empleó en Venezuela por más de un siglo marcando una pauta en la enseñanza de esta temática y sirviendo de modelo a seguir para los autores nacionales de obras didácticas de geometría. Fue texto obligado en la Academia de Matemáticas de Cagigal desde los inicios de dicho plantel y también fue adoptado por la Universidad al pasar la formación de ingenieros a esa casa de estudios. Asimismo, los Colegios Nacionales y muchos colegios privados acogieron la obra como texto de estudio para la geometría.

Particular relevancia reviste la edición realizada en 1854 por Juan de Dios Morales la cual constituye la primera edición hispanoamericana de la obra antecediendo a la bogotana de 1866 que se tenía como la primera. Adicionalmente la edición caraqueña es una reproducción de la traducción ejecutada por Gilman en 1827 de la $12^{\circ}$ original de Legendre, mientras que la colombiana se tradujo de la versión de la obra de Legende modificada por Blanchet. En lo que sí mantiene su primacía la obra publicada en Bogotá es en ser la primera traducción realizada en la América Hispana.

El análisis y comparación de los diversos ejemplares consultados ha permitido corroborar los asertos formulados en trabajos previos (p.e. García Azcárate, 2004) acerca del enfoque riguroso de la geometría (en dos y tres dimensiones) presentado en la obra, así como la existencia de dos niveles de lectura: uno establecido en el cuerpo del libro que conforma un texto de estudio para aprendices de esta área de las matemáticas; y otro, desarrollado a través de las notas dedicado a los estudios de la matemática como campo disciplinar, para los que quisieran profundizar o incursionar en la tareas investigativas. 
Con respecto a la traducción al castellano se pudo determinar que las ediciones que circularon en el país desde 1854 y antes de $1873(1854,1865)$ estuvieron basadas en la $12^{\mathrm{a}}$ edición francesa de 1823 traducida por Gilman en 1827; mientras que con respecto a la de 1841 no hay seguridad. En la edición de 1873 por vez primera aparece Jesús Muñoz Tébar vinculado a la traducción del libro. El análisis de la participación del ingeniero venezolano en esta tarea permite aseverar que fue él el traductor de las ediciones venezolanas realizadas en 1873 y a partir de allí (1879/1880, 1908/1895) basándose en la edición 15ª francesa.

Desde el punto de vista comercial la obra fue un éxito de ventas lo cual se constata en las permanentes ofertas de las casas libreras promocionando el libro en sus respectivos catálogos, en la prensa y mediante hojas sueltas (volantes). El texto fue vendido por los principales comercios del ramo en ediciones importadas (traducidas al castellano o en francés) e incluso en ediciones propias, algunas traducidas en el país. Además su difusión fue amplia llegando a diversos lugares del interior de la nación mediante la amplia red comercial que tenían algunos de los empresarios del ramo.

Entre los principales cambios y modificaciones entre una y otra edición, así como características comunes resaltantes, cabe mencionar:

- En las tres ediciones analizadas la obra consta de dos partes (Geometría y Trigonometría), editadas en un solo volumen en $8^{\circ}$ pero a veces con distinta fecha de edición entre las partes.

- El texto de la parte dedicada a la geometría está subdividido en ocho libros, siguiendo la tradición euclídea; cada libro a su vez tiene subdivisiones configurando un todo de estructura arborescente y escrito en un formato que sigue esencialmente el modelo jurídico.

- El diseño y la presentación de la obra fue mejorado por ejemplo intercalando las figuras en el texto, así como se fue revisando y mejorando la traducción del texto.

- Comparando entre sí las ediciones se aprecian ciertas diferencias: ausencia/presencia de notas y de su ubicación; ausencia/presencia de apéndices; y algunas pequeñas modificaciones en la redacción. También existe diferencias en la paginación.

Ha de concluirse señalando que los Elementos de Geometría de Legendre constituyen una obra de innegable importancia la cual cual tuvo un gran impacto en el país así como un prolongado y continuado uso en el ámbito educativo venezolano en distintos niveles, 
desplazando el uso de otros libros como los Elementos de Euclides y la contemporánea Geometría de Lacroix.

Como punto final es de reseñar que existe un conjunto de ediciones como la de 1841 o la 1865 de las cuales no se han localizado ejemplares y sólo se cuenta con escasa información que no va más allá de su anuncio en catálogos u otros medios o en breves entradas en estudios de carácter bibliográfico. Estas ediciones son las que hemos denominado "las extraviadas" y cuya localización sería de enorme interés para profundizar el tema aquí tratado.

\section{Referencias}

Albis González, V. S. (1977). Latin-American translations of Legendre's "Éléments de Géométrie". Historia Mathematica, 4(3), 339-340. Recuperado de: https://www.sciencedirect.com/journal/historia-mathematica/vol/4/issue/3.

Albis G., V. \& Sánchez B., C. E.. (s/f). Luis María Lleras, su traducción de los Éléments de Géométrie de Legendre y su correspondencia con Hermite.. Recuperado desde https://accefyn.com/microsites/grupos/historia-filosofia/wpcontent/uploads/2018/05/LuisMaraLlerasMathesis3.pdf.

Best, J. W. (1961). Cómo investigar en educación. México: Editora Nacional.

Beyer, W. (2012). Estudio evolutivo de la enseñanza de las mateméticas elementales en Venezuela a través de los textos escolares:1826-1969. La Paz, Bolivia: Instituto Internacional de Integración Convenio Andrés Bello-Grupo de Investigación y Difusión en Educación Matemática.

Beyer, W. (2019). Ediciones y traducciones venezolanas de los Éléments de Geométrie de Legendre. Un preámbulo para el estudio de su impacto en Venezuela. Revista Colombiana de Matemática Educativa (RECME), 4. (Por aparecer).

Cagigal, J. M. (1834). III Informe dado al Gobierno por el Director de la Academia de Matemáticas. En: Luis Correa (Comp.). (1956). Juan Manuel Cagigal. Escritos literarios y científicos (pp. 29-34). Caracas: Imprenta Nacional.

Cagigal, J. M. (1836). IV Informe dado al Gobierno por el Director de la Academia de Matemáticas. En: Luis Correa (Comp.). (1956). Juan Manuel Cagigal. Escritos literarios y científicos (pp. 35-40). Caracas: Imprenta Nacional.

Cagigal, J. M. (1838). VI Informe dado al Gobierno por el Director de la Academia de Matemáticas. En: Luis Correa (Comp.). (1956). Juan Manuel Cagigal. Escritos literarios y científicos (pp. 47-51). Caracas: Imprenta Nacional.

Castellanos, R. R. (2017a). Historia de las librerías en Venezuela (1607-1900). Tomo I. Caracas: Centro Nacional del Libro.

Castellanos, R. R. (2017b). Historia de las librerías en Venezuela (1607-1900). Tomo II. Caracas: Centro Nacional del Libro. 
Choppin, A. (2000). Pasado y presente de los manuales escolares. En: Julio Ruiz Berrío (Ed). (2000). La cultura escolar en Europa. Tendencias históricas emergentes (pp. 107-165). Madrid: Editorial Biblioteca Nueva.

Cilento Sarli, A.; López Villa, M. A.; Marcano González, Luis F. \& Martín Frechilla, J. J. (1999). El dispositivo de obras públicas en Venezuela (1874-1976). En: J. J. Martín Frechilla \& Texera, Y. (Comps.) (1999). Modelos para desarmar. Instituciones y disciplinas para una historia de la ciencia y la tecnología en Venezuela (pp. 49-125). Caracas: Consejo de Desarrollo Científico y Humanístico, Universidad Central de Venezuela.

Damirón y Dupouy. (1841). Catálogo de los libros y mercancías que se hallan de venta en el Almacén de Damirón y Dupouy. Caracas: Imprenta de Francisco de P. Nuñez.

École des Ponts et Chaussées (1872). Catalogue des livres composant la Bibliothèque de l'École des Ponts et Chaussées. Paris: Imprimerie Nationale. Recuperado de: https://books.google.co.ve/books?id=ubE_AQAAMAAJ\&pg=PR1\&lpg=PR1\&dq=\%22Cat alogue+des+livres+composant+la+Biblioth\%C3\%A8que+de+1\%27\%C3\%89cole+des+Pon ts+et+Chauss \%C3\%A9es\%22\&source=bl\&ots=WsNtN0VoUq\&sig=ACfU3U3PrdojufFa GBw7W2f8dMeLTNyag\&hl=es-419\&sa=X\&ved=2ahUKEwiJzuv1qcvoAhVKT98KHY8CoU4ChDoATAAegQICxAp\#v=onepage $\& q=\% 22$ Catalogue $\% 20$ des $\% 20$ livres $\% 20$ compos ant $\% 201 \mathrm{a} \% 20$ Biblioth\%C3\%A8que $\% 20 \mathrm{de} \% 201 \% \mathrm{C} 3 \% 89$ cole $\% 20 \mathrm{des} \% 20$ Ponts $\% 20 \mathrm{et} \% 20$ Chauss\%C3\%A9es\%22\&f=false.

Ernst, A. (1875). Catálogo de la biblioteca de la Universidad de Caracas. Formado de orden del Ilustre Americano, Regenerador y Presidente de los Estados Unidos de Venezuela,General Guzmán Blanco. Caracas: Imprenta de "La Opinión Nacional”.

Freytes, Y. (2000). Un esbozo histórico de las matemáticas en Venezuela. I Parte: Desde la Colonia hasta finales del siglo XIX. Boletín de la Asociación Matemática Venezolana, 7(12), 9-37. Recuperado de: https://www.emis.de/journals/BAMV/conten/vol07/yfreites.pdf.

Frydensberg, A. (1895). Materiales para la bibliografía nacional. En: R. Seijas (Ed.). (1974). Primer libro venezolano de literatura, ciencias y bellas artes (pp. 304-336). Caracas: Concejo Municipal del Distrito Federal.

García Azcárate, A. (2004). Un best-seller del siglo XIX: Los Elementos de Geometría de Legendre. En: J. J. Escribano Benito et al. (Eds.). Actas VIII Congreso de la Sociedad Española de Historia de las Ciencias y de las Técnicas. Logroño: SEHCYT-Universidad de La Rioja, 357-367. Recuperado de: https://docplayer.es/28530187-Un-best-seller-del-sigloxix-los-elementos-de-geometria-de-legendre-ana-garcia-azcarate.html.

Gimeno Sacristán, J. (1998). El curriculum: una reflexión sobre la práctica. Madrid: Ediciones Morata.

Legendre, A. M. (1823). Éléments de Géométrie, avec des notes; par A. M. Legendre, membre de l'Institut et de la Légion d'Honneur, de la Société Royale de Londres, etc. Paris: Firmin Didor père et fils. Recuperado de: https://gallica.bnf.fr/ark:/12148/bpt6k9771899n/f9.image.

Legendre, A. M. (1854a). Elementos de Geometría escritos en francés por A. M. Legendre, individuo del Instituto de Francia, de la Real Sociedad de Londres \& c. Traducidos por la duodécima y última edición. Caracas: Imprenta de Juan de Dios Morales. 
Walter O. Beyer K.

Legendre, A. M. (1854b). Elementos de Trigonometría escritos en francés por A. M. Legendre, individuo del Instituto de Francia, de la Real Sociedad de Londres \& c. Traducidos por la duodécima y última edición. Caracas: Imprenta de Juan de Dios Morales.

Legendre, A. M. (1862). Éléments de Géométrie, avec des notes; par A. M. Legendre, membre de l'Institut et de la Légion d'Honneur, de la Société Royale de Londres, etc. Paris: Firmin Didor fréres, fils et $c^{\text {ie }}$. Recuperado de: https://ia800304.us.archive.org/26/items/lmentsdego00lege/lmentsdego00lege.pdf.

Legendre, A. M. (1879). Elementos de geometría (con notas). Escritos en francés por A. M. Legendre, miembro del Instituto de Francia, de la Legión de Honor, de la Real Sociedad de Londres \& c. Traducidos por la décima y quinta edición. Revisada por el Dr. Jesús Muñoz. Tébar-Ingeniero. Caracas: Alfred Rothe-Editor.

Legendre, A. M. (1880). Elementos de trigonometría. Escritos en francés por A. M. Legendre, miembro del Instituto de Francia, de la Legión de Honor, de la Real Sociedad de Londres \& c. Traducidos por la décima y quinta edición. Revisada por el Dr. Jesús Muñoz TébarIngeniero. Caracas: Alfred Rothe-Editor.

Legendre, A. M. (1895). Elementos de trigonometría. Escritos en francés por A. M. Legendre. Miembro del Instituto de Francia, de la Legión de Honor, de la Real Sociedad de Londres, etc. Última edición. Figuras intercaladas en el texto. Revisada por el Dr. Jesús Muñoz Tébar-Ingeniero. Caracas: L. Puig Ros y Hermano.

Legendre, A. M. (1908). Elementos de geometría (con notas). Escritos en francés por A. M. Legendre. Miembro del Instituto de Francia, de la Legión de Honor, de la Real Sociedad de Londres, etc. Última edición. Figuras intercaladas en el texto. Revisada por el Dr. Jesús Muñoz Tébar-Ingeniero. Caracas: Librería Española de L. Puig Ros.

Ministerio de Obras Públicas. (1875). Memoria del Ministerio de Obras Públicas al Congreso de los Estados Unidos de Venezuela, en 1875. Caracas, Imprenta Federal. Recuperado de: https://books.google.co.ve/books?id=BUBRAQAAMAAJ\&pg=PA12\&lpg=PA12\&dq=\%2 $\underline{2 \text { sus }+ \text { traducciones }+ \text { de }+ \text { los }+ \text { libros }+ \text { de }+ \text { Lacroix }+y+d e+\text { Legendre } \% 22 \& \text { source }=\text { bl\&ots }=51 \mathrm{nz}}$ taqVj0\&sig=ACfU3U0Sbq8iWUZKNwuHeFnjs2eT06xvsQ\&hl=es\&sa=X\&ved=2ahUKE wjE8Kal3c3oAhWrnOAKHeTbDrwQ6AEwAHoECAIQKQ\#v=onepage\&q=\%22sus\%20tr aducciones $\% 20 \mathrm{de} \% 201 \mathrm{los} \% 20$ libros $\% 20 \mathrm{de} \% 20$ Lacroix $\% 20 \mathrm{y} \% 20 \mathrm{de} \% 20$ Legendre $\% 22 \& \mathrm{f}=\mathrm{f}$ alse.

Museo del Transporte Guillermo José Schael. (2014). Librerías y Libreros de Caracas. Recuperado de: http://museodeltransportecaracas.blogspot.com/2014/10/librerias-ylibreros-de-caracas.html.

Olivar, J. A. (2008). Jesús Muñoz Tébar. Caracas: C. A. Editora El Nacional-Bancaribe.

Pardo Stolk, E. \& de Amezaga, V. (1973). Jesús Muñoz Tébar. Caracas: Ministerio de Educación, Dirección General, Departamento de Publicaciones. 
Preveraud, T. (2013). Transmissions des enseignements mathématiques français à l'Académie militaire américaine de West Point (1815-1836). Amnis, 12. Recuperado desde http://journals.openedition.org/amnis/1943.

Rothe, A. (1880). Otras obras publicadas en la misma Casa. En: A. Gossart (1880). Estenaritmia o abreviación de los cálculos, complemento indispensable de la aritmética. Caracas: Allfred Rothe Editor.

Sánchez, M. S. (1946). Bibliografía de obras didácticas publicadas en Venezuela o por autores venezolanos en el exterior. Caracas: Tipografía Americana.

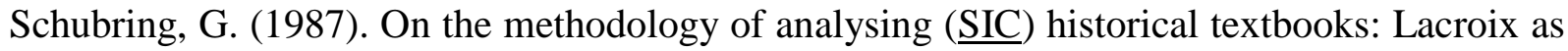
textbook author. For the Learning of Mathematics, 7(3), 41-51.

Schubring, G. (2003). Análise histórica de livros de matemática. Campinas: Editora Autores Associados.

Schubring, G. (2009). A origem da geometria de Legendre e o seu impacto international. En: Luis Carlos Guimarães (Org.). Adrien Marie Legendre, Elementos de Geometria. Reediçao da primera traduçao brasileira de 1809 (pp. 353-384). Rio de Janeiro: Editotra LIMC.

Zawisa, L. (1980). La Academia Matemática de Caracas. Caracas; Ministerio de la Defensa.

Zawisa, L. (1988). Arquitectura y obras públicas en Venezuela. Siglo XIX. Tomo I. Caracas: Ediciones de la Presidencia de la República. 